\title{
Quantum phase transitions and new scales in QCD-like theories
}

\author{
Mithat Ünsal \\ SLAC and Department of Physics, \\ Stanford University, CA, 94309/94305
}

(Dated: January 11, 2021)

\begin{abstract}
It is commonly believed that in confining vector-like gauge theories the center and chiral symmetry realizations are parametrically entangled, and if phase transitions occur, they must take place around the strong scale $\Lambda^{-1}$ of the gauge theory. We demonstrate that (non-thermal) vector-like theories formulated on $\mathbb{R}^{3} \times S^{1}$ where $S^{1}$ is a spatial circle exhibit new dynamical scales and new phenomena. There are chiral phase transitions taking place at $\Lambda^{-1} / N_{c}$ in the absence of any change in center symmetry. $\Lambda^{-1} / N_{c}$, invisible in (planar) perturbation theory, is also the scale where abelian versus non-abelian confinement regimes meet. Large $N_{c}$ volume independence (a working Eguchi-Kawai reduction) provides new insights and independently confirms the existence of these scales. We show that certain phases and scales are outside the reach of holographic (supergravity) modeling of QCD.
\end{abstract}

PACS numbers: 12.38.Aw, 11.15.Ex, 11.15.Tk, 11.30.Rd

\section{QUANTUM PHASE TRANSITIONS IN QCD ON $\mathbb{R}^{2,1} \times S^{1}$}

Consider an asymptotically free, confining QCD-like gauge theory with a rank $N_{c}$ gauge group $G$ and $n_{f}$ flavors of massless fermions in a vector-like representation $\mathcal{R}$ of $G$ (or a mixture of representations), formulated on a space with one compact dimension. Such theories possess a strong dynamical scale $\Lambda$ as a consequence of dimensional transmutation.

There are three pieces of conventional wisdom associated with this class of theories. a) Even though the chiral symmetry and center symmetry are independent symmetries, their realization are parametrically entangled. b) If phase transitions occur, they must take place in the numerical vicinity of strong scale $\Lambda$. c) If one takes the large $N_{c}$ limit (in the conventional 't Hooft sense [1]), the scale of the phase transitions must be $O\left(N_{c}^{0}\right)$.

Lattice gauge theory simulations unambiguously demonstrate that in finite temperature phase transitions, the above conventional wisdom is correct. Any confining QCD-like theory, regardless of the representation $\mathcal{R}$ of the fermions, will undergo a center symmetry (or approximate center symmetry if center symmetry is absent) changing confinement-deconfinement transition accompanied with the chiral symmetry transition which occurs around the strong scale $\Lambda[2,3$. The fact that a very high temperature phase with broken center symmetry cannot support any kind of chiral condensate can be proven rigorously [4. The second assertion seems to be robust due to the absence of any other dimensionful parameters in the theory. The last one states that the large $N_{c}$ limit should be a good guide to probe such transitions. This is also an underlying assumption in recent holographic models of QCD. The thermal QCD-like theories provide full support for these three assertions.

In this letter, we wish to examine the validity of these common assertions by testing them in a slightly different setup. We wish to classify the phases of zero temperature
QCD-like theories on a space with one compact spatial dimension, $\mathbb{R}^{2,1} \times S^{1}$, as a function of $S^{1}$ radius. We will work in a Euclidean setup, $\mathbb{R}^{3} \times S^{1}$, hence the antiperiodic or periodic spin structure of the fermions, $\mathcal{S}^{\mp}$, along the $S^{1}$ circle, determines whether $S^{1}$ is thermal or spatial circle, respectively.

The rationale behind this proposal lies in the sharp qualitative differences between thermal and quantum fluctuations. In this sense, using periodic boundary conditions for fermions is equally physical, and in some ways a better guide to single out the quantum fluctuations. The phase transitions on spatial $S^{1} \times \mathbb{R}^{3}$ (if any), are induced by zero temperature quantum mechanical fluctuations rather then the thermal fluctuations. Hence these are quantum phase transitions, as often appear in condensed matter physics [5].

These quantum phase transitions reveal new and surprising phenomena in clear contradiction with the conventional wisdom. Most notably, there are cases with chiral transitions in the absence of any change in center symmetry realizations. More interestingly, some such transitions takes place at a scale parametrically split from $\Lambda^{-1}$ by factors of $N_{c}$. For example, in QCD(adj) with multiple fermions and periodic boundary conditions (in which center symmetry never breaks [6]), we will show that the chiral transition scale is

$$
L_{\chi}=c \Lambda^{-1} / N_{c}
$$

where $c$ is an order one numerical factor. The existence of the scale (1) is the main new result of this letter. This scale is invisible in the planar perturbation theory which combines the two coupling $\left(g^{2}, N_{c}\right)$ into a single 't Hooft coupling $\lambda=g^{2} N_{c}$ [1]. The reason is, the planar perturbative expansion does not respect the center symmetry of the small $S^{1}$ regime.

The presence of such a suppressed scale may seem exotic. However, I will argue that it must exist in all confining QCD-like theories in the following sense: Were the center symmetry to remain unbroken in a multi-flavor 
QCD-like theory (either thermally or spatially compactified), a chiral transition would still take place and it would occur at $c \Lambda^{-1} / N_{c}$ (and not at $\left.\Lambda^{-1}\right)$. Hence, 11 is in fact the natural scale of the chiral transition in QCD, answering a question raised in [7.

\section{A. Classification}

The vector-like gauge theories with massless fermions formulated on $\mathbb{R}^{3} \times S^{1}$ split into at least four categories according to their spatial center $\left(C_{s}\right)$, and chiral $(\chi)$ symmetry realizations. The classes are:

i) The theories in which $C_{s}$ and $\chi$ symmetry realizations are entangled, either into a single transition or a non-parametrically separable double transition, and both transitions occur around strong scale $L_{c} \sim \Lambda^{-1}$.

ii) The theories without any phase transitions

iii) Center symmetric theories with a chiral transition at a suppressed scale $L_{\chi} \sim \Lambda^{-1} / N_{c}$.

iv) The theories in which $\chi$ and $C_{s}$ symmetries are entangled, and both transitions occur around a suppressed scale $L_{c} \sim \Lambda^{-1} / N_{c}^{k}$, for some $k>0$.

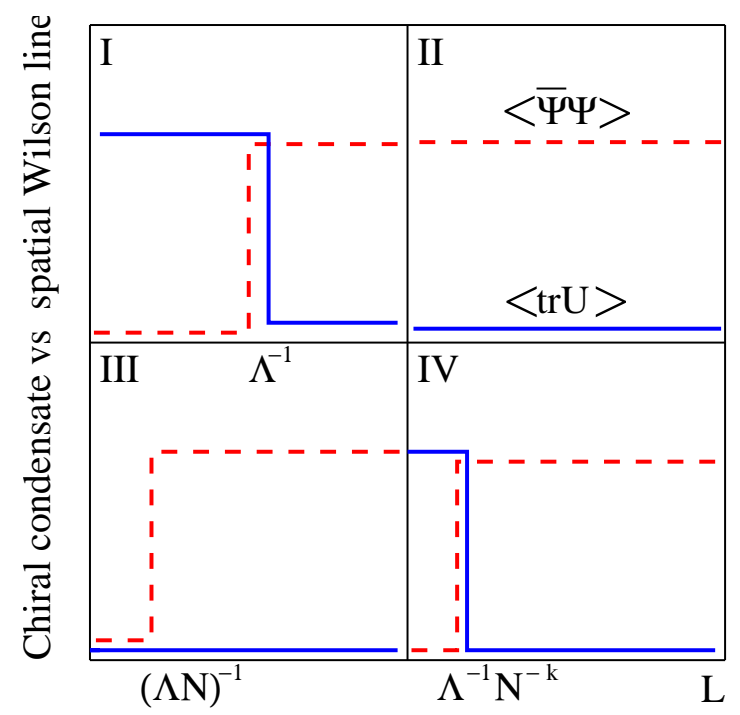

FIG. 1: The cartoons of the zero temperature center (blue, straight) and chiral (red, dotted) symmetry realizations in vector-like theories with periodic boundary conditions for fermions. In the thermal case, only class I) is possible.

As asserted earlier, this richness is a reflection of the distinction of quantum versus thermal fluctuations. Sufficiently high temperatures melt the hadrons and glueballs into quarks and gluons regardless of the fermionic matter content of the theory. The quantum fluctuations may or may not generate quantum melting. This depends on the matter content of the theory. Therefore, probing a theory solely based on quantum fluctuations may be a better guide to study QCD dynamics.

A corollary of classification: At $N_{c}=\infty$, iii) and iv) degenerate into ii). This means, the critical radius of transition for the $N_{c}=\infty$ theory is $L_{c}=0$.

Phase transition as a function of $N_{c}$ : The corollary implies that, although an $S U(3)$ gauge theory on small, fixed $S^{1} \times \mathbb{R}^{3}$ may be in a confinement without chiral symmetry breaking phase, on the same four-manifold, the $S U(\infty)$ theory is in a chirally asymmetric, confinement phase. In other words, the large $N_{c}$ limit is separated from the small $N_{c}$ theory on the same four-manifold by a phase transition. In such circumstances, the large $N_{c}$ is not a good guide to study phases of the small $N_{c}$ theories, and such cases are generic.

\section{B. Applications}

We assume the vector-like theories of our interest are in center symmetry unbroken, chiral symmetry broken phase at large $S^{1} \times \mathbb{R}^{3}$. In the small $S^{1}$ regime, the center symmetry realization can be determined by conventional techniques, by evaluating the one loop potential in the background of the constant spatial Wilson line $U(x)=P e^{i \int A_{4}\left(x, x_{4}\right) d x_{4}}$, and by using periodic boundary conditions for fermions. Below are the simplest examples for the four classes.

i) Consider QCD with $n_{f}$ complex representation fermions, such as fundamental, antisymmetric or symmetric. In these theories, the examination of the effective potential $V_{\text {eff }}[U(x)]$ on sufficiently small $S^{1}$ shows that $\langle\operatorname{tr} U(x)\rangle \neq 0$, and center symmetry is broken. The effect is due to $O\left(N_{c}^{2}\right)$ part of the potential. The perturbative one loop potential also renders fermions massive with a gap of order $\frac{1}{L}$. Hence, the long distance correlators of chiral operators decays exponentially, with no formation of any condensate. Although this class do not exhibit any novel scales, the center broken phase also breaks C, P, T as well as CPT. 8]. In this sense, it is an interesting phase. The lattice studies of such QCD-like theories with one compact spatial dimension is initiated recently in Refs. 9, 10, and for $S U(3)$ gauge theory, a CPT breaking and restoring transition is observed.

ii) The examples of this class are $\mathcal{N}=1 \mathrm{SYM}$ theory with arbitrary gauge group $G$. The one loop potential is $V_{\text {eff }}[U]=0$ due to supersymmetry. Nonetheless, a nonperturbatively generated potential provides a repulsive interaction among eigenvalues of the spatial Wilson line, and the spatial center symmetry $C_{s}$ is unbroken.

The unbroken $C_{s}$ in the weakly coupled regime implies gauge symmetry "breaking" down to the maximal abelian subgroup, $G \rightarrow \mathbf{A b}(G)$ at large distances. Consequently, the fermion modes along the Cartan subalgebra of $G$ remains massless, and they are part of the long distance physics. The fractional instanton (monopole) effects are sufficient to produce a chiral condensate and 
spontaneous breaking of discrete chiral symmetry. The IR dynamics in this case is drastically different from both case i) and the thermal compactification. Small $S^{1}$ exhibits abelian confinement and discrete chiral symmetry breaking [12, 13, 14.

iii) The asymptotically free QCD-like theories with $n_{f}>1$ adjoint Majorana fermion are in this category. Introducing adjoint fermions with periodic boundary conditions $\mathcal{S}^{+}$stabilizes the center symmetry breaking instability [6]. The one loop potential for QCD(adj) with $\mathcal{S}^{+}$is proportional to the negation of the one of pure YM theory:

$$
V_{\mathrm{eff},+}^{\mathrm{QCD}(\operatorname{adj})}[U(x)]=\left(1-n_{f}\right) V_{\mathrm{eff}}^{\mathrm{YM}}[U(x)]
$$

This implies unbroken spatial center symmetry in QCD(adj) with $\mathcal{S}^{+}$at small $S^{1}$ weak coupling regime, and consequent dynamical abelianization down to $\mathbf{A b}(G)$ at large distances. $\mathrm{QCD}(\mathrm{adj})$ has a continuous $S U\left(n_{f}\right)$ chiral symmetry. At small $S^{1}$, the theory exhibits confinement without chiral symmetry breaking as shown in 14. for $N_{c}=$ few. At long distances, abelian confinement is operative just like the $\mathcal{N}=1$ SYM theory in the same regime, and the photons acquire mass via magnetic bion mechanism. At length scales larger than the inverse photon mass, the long distance effective theory reduce to a NJL-type Lagrangian of zero mode fermions which is known to possess two phases: a weak coupling unbroken phase and a strong coupling broken phase. Although the strong coupling phase is outside the region of validity of the effective theory, the transition is taking place just at its boundary where $e^{-S_{0}} \sim 1$. Here, $S_{0}=\frac{8 \pi^{2}}{g^{2}\left(m_{W}\right) N_{c}}$ is the monopole (fractional instanton) action.

The interesting aspect, which is not addressed in [14] in generality, is that in terms of compactification radius $L, N_{c}$ and $\Lambda$, this translates into $L N_{c} \Lambda \sim 1$. This is also the scale where the non-perturbatively generated photon mass becomes equal to the lightest $W$-boson mass $m_{W}=2 \pi /\left(N_{c} L\right)$ and a long distance description based on $\mathbf{A b}(G)$ ceases its validity. The theory moves from an the abelian confinement to non-abelian confinement regime [15. In multi-flavor $\mathrm{QCD}(\operatorname{adj})$ theory, this is associated with a solo chiral phase transition.

There is a deeper reason for the existence of a suppressed chiral transition scale $\Lambda^{-1} / N_{c}$. The nonperturbative physics of large $N_{c}$ QCD-like gauge theory is independent of the volume of the $S^{1}$ so long as the center symmetry is unbroken [16, 17, 18. This is the volume independence property at large $N_{c}$, also known as EguchiKawai reduction. For pure YM, a full EK reduction fails just because the center symmetry breaks spontaneously. $\mathrm{QCD}(\operatorname{adj})$ with $\mathcal{S}^{+}$is the first continuum gauge theory example which satisfies the volume independence all the way down to arbitrarily small volumes [6], as opposed to the partial reduction discussed in [19] and other schemes which are recently shown to fail [20, 21, 22].

The independence of the physics from the $S^{1}$ size at $N_{c}=\infty$ limit implies that the chiral symmetry realization and chiral condensate must be independent of $S^{1}$ radius in $\mathrm{QCD}(\operatorname{adj})$. Therefore, whatever chiral transition takes place at finite $N_{c}$ and finite $S^{1}$ must be pushed to $L_{\chi}=0$ at $N_{c}=\infty$. Also note that the existence of $\mathbf{A b}(G)$ regime [14] and the large $N_{c}$ volume independence [6] are consistent with each other, because the region of validity of $\mathbf{A b}(G)$ regime is pushed into an arbitrarily narrow sliver as $N_{c}$ is taken large.

iv) The YM theory with one adjoint Majorana and $n_{f} \geq 1$ fundamental Dirac fermions all with $\mathcal{S}^{+}$is in this class. For $n_{f}=0$, the theory is just $\mathcal{N}=1$ SYM. Due to supersymmetry of $n_{f}=0$ background, $V_{\text {eff }}[U]$ is $0 \times O\left(N_{c}^{2}\right)+n_{f} O\left(N_{c}\right)$ where the first non-vanishing contribution is due to $n_{f}$ fundamental fermions. The minimum of the $V_{\text {eff }}[U]$ is at $U=-1$ and the center is broken at small $S^{1}$. The interesting behavior of this theory is due to the fact that the one loop potential is $n_{f} O\left(N_{c}\right)$. Recall from thermal QCD -the case in which thermal one-loop potential is $O\left(N_{c}^{2}\right)$ - that the usual electric mass of the holonomy (or the $A_{4}$ field) is $m_{e} \sim \frac{\sqrt{\lambda}}{L}$. In our case, the mass of the $A_{4}$ field is anomalously small [23]

$$
m^{*}=\frac{\sqrt{\lambda}}{L} \sqrt{\frac{n_{f}}{N_{c}}} \equiv m_{e} \sqrt{\frac{n_{f}}{N_{c}}}
$$

In the large $N_{c}$ limit, $m^{*} / m_{e} \rightarrow 0$ and the one loop effective potential seen by the $N_{c}$ eigenvalues becomes arbitrarily flat. A classical moduli space opens up at this level of analysis. As in the $\mathcal{N}=1 \mathrm{SYM}$ case, the non-perturbative potential restores the center symmetry. This means, the chiral and center symmetry transition scales must be suppressed scales relative to $\Lambda^{-1}$.

Another way to realize that there must exist a suppressed chiral and center symmetry transition scale is to use the large $N_{c}$ volume independence. In the $N_{c}=\infty$ limit, the SYM theory obeys volume independence. The theory on $\mathbb{R}^{4}$ is nonperturbatively equivalent to the theory on $\mathbb{R}^{3} \times S^{1}$ for any finite $S^{1}$. In other words, (somehow counter-intuitively), the $N_{c}=\infty$ limit of $\mathcal{N}=1$ SYM lacks a weak coupling $\mathbf{A b}(G)$ description at long distances regardless of how small $S^{1}$ is, so long as it is finite. The addition of fundamental fermions on small $S^{1} \times \mathbb{R}^{3}$ is same as adding fundamental fermions to the theory on $\mathbb{R}^{4}$. Consequently, at $N_{c}=\infty$ limit, fundamental fermions cannot induce a center symmetry breaking in this theory.

\section{Implications and comments}

Refined (abelian) large $N_{c}$ limit: For any confining QCD-like gauge theory which remains center symmetric at arbitrarily small $S^{1} \times \mathbb{R}^{3}$, there exists a double-scaled, refined large $N_{c}$ limit. In this limit,

$$
\Lambda^{-1}=O\left(N_{c}^{0}\right), \quad L N_{c} \Lambda=O\left(N_{c}^{0}\right) \ll 1
$$

are held fixed. The short distance is $U(\infty)$ and long distance gauge structure is a $[U(1)]^{\infty}$ mimicking the $\mathbf{A b}(G)$ 
structure of the small $N_{c}$, small $S^{1}$ center symmetric theories. The existence of an abelian large $N_{c}$ limit was first shown in non-vector-like $\mathcal{N}=2$ SYM theory [25]. Realizing that such a limit exists and is generic in QCD-like theories is new. We expect the refined large $N_{c}$ limits to be generically solvable as in 24.

A no-go theorem for holographic (supergravity) models of QCD: Since supergravity is the classical $N_{c}=\infty$ limit, the small radius phases of the classes iii) and iv) are invisible in the holographic modeling of QCD as a result of corollary. In particular, the confinement without chiral symmetry breaking phase and the associated solo chiral transitions are outside the reach of supergravity approximation. A stringy improvement of holographic models, which incorporates the $O\left(1 / N_{c}\right)$ effects, is needed to find the phase transitions in classes iii) and iv).

Confirming this result, the holographic models of QCD so far did not find any confinement without chiral symmetry breaking phase, although chirally asymmetric deconfined phases were found [26, 27]. This non-observation can naturally be explained by large $N_{c}$ volume independence. It is also tied with "dynamical abelian dominance" of the refined large $N_{c}$ limit, one cannot describe phases with $O\left(N_{c}\right)$ weakly coupled fields as in [11, 24, 25] in supergravity approximation [23].

Testing on the lattice: One may wonder why these novel scales are not already seen in lattice gauge theory simulations. The reason is simple. So far, there exist no non-thermal lattice simulation for classes iii) and iv).

Even in the thermal setting, the confinement without chiral symmetry breaking phase can be observed as follows: Assume the center symmetry is stabilized at small $S^{1}$ by a double trace deformation $[11,24$. In multi-flavor theories, there will still be a solo chiral transition from a confined chirally asymmetric to a confined chirally symmetric phase, which will take place at $\beta_{\chi}=c \Lambda^{-1} / N_{c}$ due to similar reasons as in $\mathrm{QCD}(\mathrm{adj})$. In this sense, the natural scale of chiral transition is again (1). In the thermal QCD, this scale is shadowed by the deconfinement transition which occurs around $\Lambda^{-1}$ and forces the chiral symmetry to restore 4 far before $\Lambda^{-1} / N_{c}$. The simulation of deformed QCD and the quantum phase transitions are feasible by conventional techniques.

Summary: In any confining QCD-like theory which always remains center symmetric (either by non-thermal fluctuations or by deformations) on $\mathbb{R}^{3} \times S^{1}$, the scale $\Lambda^{-1} / N_{c}$ is the most important scale. For smaller radius, the long distance theory abelianizes down to $\mathbf{A b}(G)$ and abelian confinement is operative. For larger radius, nonabelian confinement is valid. For zero and one flavor theories, the abelian and non-abelian confinement regimes are smoothly connected [11, 24]. For multi-flavor theories, there must exist a single chiral transition from a chirally symmetric abelian confinement regime to a chirally asymmetric nonabelian confinement regime occurring at $c \Lambda^{-1} / N_{c}$. Since the non-abelian confinement and volume independence holds for $L N_{c} \Lambda>1$, and the theory just above this critical radius is equivalent to QCD on $\mathbb{R}^{4}$ up to $O\left(1 / N_{c}^{2}\right)$ corrections, understanding the dynamics of QCD in the vicinity of (1) may hold the necessary insights into a fuller understanding of the theory.

Acknowledgments: I am grateful to O.Aharony, R. Brower, D. Harlow, D.Kutasov, A. Parnachev, M. Shifman and L. Yaffe for useful discussions. This work is supported by the U.S. Department of Energy Grant DEAC02-76SF00515.
[1] G. 't Hooft, Nucl. Phys. B 72, 461 (1974).

2] M. Cheng et al., Phys. Rev. D 77, 014511 (2008) arXiv:0710.0354 [hep-lat]].

[3] Y. Áki, G. Endrodi, Z. Fodor, S. D. Katz and K. K. Szabo, Nature 443, 675 (2006) arXiv:hep-lat/0611014.

[4] E. T. Tomboulis and L. G. Yaffe, Phys. Rev. Lett. 52, 2115 (1984).

[5] S. Sachdev, Quantum phase transitions, Cambridge, 1999

6] P. Kovtun, M. Unsal and L. G. Yaffe, JHEP 0706, 019 (2007) arXiv:hep-th/0702021.

[7] A. Mocsy, F. Sannino and K. Tuominen, Phys. Rev. Lett. 92, 182302 (2004) arXiv:hep-ph/0308135.

[8] M. Ünsal and L. G. Yaffe, Phys. Rev. D 74, 105019 (2006) arXiv:hep-th/0608180.

[9] 'T. DeGrand and K. Hoffmann, JHEP 0702, 022 (2007) arXiv:hep-lat/0612012.

[10] B. Lucini, A. Patelia and C. Pica, Phys. Rev. D 75, 121701 (2007) arXiv:hep-th/0702167.

111 M. Shifman and M. Ünsal, arXiv:0802.1232 [hep-th].

[12] S. H. Katz and C. Vafa, Nuci. Phys. B 4977, 196 (1997) arXiv:hep-th/9611090.

[13] N. IM. Davies, T. J. Hollowood and V. V. Khoze, J. Math. Phys. 44, 3640 (2003) arXiv:hep-th/0006011.
[14] M. Unsal, arXiv:0709.3269 [hep-th].

15. M. Shifman and A. Yung, Phys. Rev. D 77, 066008 (2008) arXiv:0712.3512 [hep-th]].

[16] 'T. Eguchi and H. Kawai, Phys. Rev. Lett. 48, 1063 (1982).

[17] L. G. Yaffe, Rev. Mod. Phys. 54, 407 (1982).

[18] G. Bhanot, U. M. Heller and H. Neuberger, Phys. Lett. B 113, 47 (1982).

[19] R. Narayanan and H. Neuberger, Phys. Rev. Lett. 91, 081601 (2003) arXiv:hep-lat/0303023.

[20] M. Teper and H. Vairinhos, Phys. Lett. B 652, 359 (2007) arXiv:hep-th/0612097.

[21] 'T. Azeyanagi, Mi. Hanada, T. Hirata and T. Ishikawa, JHEP 0801, 025 (2008) arXiv:0711.1925 [hep-lat]].

[22] B. Bringoltz and S. R. Sharpe, arXiv:0805.2146 [hep-lat].

23 O. Aharony, Private communications,

24. M. Unsal and L. G. Yaffe, arXiv:0803.0344 [hep-th].

25] M. R. Douglas and S. H. Shenker, Nuci. Phys. B 447 , 271 (1995) arXiv:hep-th/9503163.

[26] O. Aharony, J. Sonnenschein and S. Yankielowicz, Annals Phys. 322, 1420 (2007) arXiv:hep-th/0604161.

[27] A. Parnachev and D. A. Sahakyan, Phys. Kev. Lett. 97, 111601 (2006) arXiv:hep-th/0604173. 